\title{
Spontaneous Fungal Peritonitis in Patients with Liver Cirrhosis
}

\author{
Toru Shizuma* \\ Department of Physiology, School of Medicine, Tokai University, Japan
}

\begin{abstract}
Although spontaneous peritonitis is a common infection in liver cirrhosis patients, little is known about the clinical characteristics and treatments of spontaneous fungal peritonitis (SFP) or fungiascites. Although diagnosis of SFP or fungiascites is made according to the cell counts and culture in ascitic fluid, delayed diagnosis has been associated with poor prognosis of SFP. Risk factors for SFP include severe underlying liver dysfunction, hospitalization and nosocomial infections. SFP mortality rates have been estimated as higher than those of spontaneous bacterial peritonitis. Although early administration of appropriate antifungal agents may be necessary, it remains uncertain whether antifungal therapies would decrease SFP's mortality rate.

Keywords: Spontaneous fungal peritonitis; Fungiascites; Spontaneous bacterial peritonitis; Liver cirrhosis

Abbreviations: Cr: Creatinine; LC: Liver Cirrhosis; MELD: Model for End-Stage Liver Disease; PCR: Polymerase Chain Reaction; PMNs: Polymorphonuclear Leukocytes; PT: Prothrombin Time; SBP: Spontaneous Bacterial Peritonitis; SFP: Spontaneous Fungal Peritonitis; spp.: species
\end{abstract}

\section{INTRODUCTION}

Patients with liver cirrhosis (LC) are susceptible to bacterial and fungal infections, which are often life-threatening complications for them [1,2]. Patients with ascites are at risk of spontaneous peritonitis due to bacterial infections (spontaneous bacterial peritonitis (SBP) and bacterascites), fungal infections (spontaneous fungal peritonitis (SFP), and fungiascites (or fungal ascites)) [3-5]. Although the clinical characteristics and prognosis of SBP have been well documented [5], the clinical characteristics of SFP and fungiascites in patients with LC are less well known. However, some clinical studies of SFP and fungiascites have been reported in recent years, including those that have evaluated and compared SFP and SBP [6-10] or spontaneous peritonitis with a funguspositive ascitic culture (SFP or fungiascites) and spontaneous peritonitis with a bacteria-positive ascitic culture (culture-positive SBP or bacterascites) $[11,12]$. In the present article, we review and summarize spontaneous peritonitis due to fungal infections (SFP and fungiascites).

\section{Diagnosis of SFP and fungiascites}

Spontaneous peritonitis is a peritoneal cavity infection with no surgically treatable intra-abdominal source of infection or malignancy [13]. Spontaneous peritonitis with culture-positive ascites is defined as culture-positive SBP and bacterascites or SFP and fungiascites. SFP is diagnosed if $\geq 250$ polymorphonuclear leukocytes (PMNs) $/ \mathrm{mm}^{3}$ are present in the ascitic fluid and the ascites are fungal-culture-positive regardless of bacterial cocolonization $[5,10]$. Fungiascites are defined as a fungus-positive ascitic culture regardless of bacterial co-colonization and $<250$ $\mathrm{PMNs} / \mathrm{mm}^{3}$ in the ascitic fluid $[5,10]$.

Early diagnosis of SFP and fungiascites is challenging, partly because of the time required for diagnostic fungal cultures [14] and partly because of lack of suspicion of SFP or fungiascites by the clinician. Diagnostic methods for SFP or fungiascites include polymerase chain reaction (PCR), 1,3-beta-D-glucan assay and galactomannan of the ascitic fluid [9,14-18].

Recent review article supports the usefulness of testing ascitic fluid using methods such as pan-fungal PCR and 1,3-beta-D-glucan assay for early diagnosis of SFP [19]. Therefore, these tests may also be performed at the first paracentesis in cases of suspected spontaneous peritonitis. Biomarkers, such as procalcitonin level and leukocyte esterase reagent strips, can help diagnose SBP [20] but may not distinguish between SFP and SBP.

\section{Fungal infections in patients with LC}

A recent multicenter study reported that of 1052 patients with LC and concomitant infections, 134 (12.7\%) involved fungal agents [3]. Prophylactic use of antibiotics and nosocomial infection both affect the incidence of fungal infection in LC [3,21]. Zhao et al. [22] reported that, of 232 episodes of bloodstream infections in

Correspondence to: Toru Shizuma, Department of Physiology, School of Medicine, Tokai University, 143, Shimokasuya, Isehara, Kanagawa 259-1193, Japan, Tel: +81-0463-93-1121; E-mail: shizuma@is.icc.u-tokai.ac.jp

Received: June 21, 2019, Accepted: August 26, 2019, Published: September 2, 2019

Citation: Shizuma T (2019) Spontaneous Fungal Peritonitis in Patients with Liver Cirrhosis. J Liver 8: 238. doi: 10.35248/2167-0889.19.8.238.

Copyright: ( 2019 Shizuma T. This is an open-access article distributed under the terms of the Creative Commons Attribution License, which permits unrestricted use, distribution, and reproduction in any medium, provided the original author and source are credited. 
patients with LC, $12(5.2 \%)$ involved fungal agents (nine were Candida albicans and three were Cryptococcus neoformans).

Underlying liver dysfunction, renal dysfunction and low ascites protein concentration reportedly increase the risk of mortality in patients with LC and fungal infections [3]. Alexopoulou et al. [8] reported that fungal infections had a significantly higher mortality rate than bacterial infections in those with LC.

\section{Spontaneous peritonitis due to fungi}

The breakdown of the intestinal mucosa barrier, dysbiosis (changes in the composition of the intestinal bacterial flora), translocation of pathogens, in addition to deficiencies in local host immune defenses may be involved in the onset of ascitic infections that reach the systemic circulation from the gut in patients with LC [23-25]. Fungi in the ascitic fluid in SFP or fungiascites may also be the result of a fundamental pathogenic mechanism of fungal translocation from the gut to systemic circulation [5,19]. Antibiotic treatment of SBP may increase the risk of fungal peritonitis by allowing excessive fungal growth in the intestinal flora [19]. Subsequent translocation of fungi into the peritoneal cavity could induce SFP or fungiascites $[19,26]$, even though their size may make fungal translocation across the gut mucosa more difficult than bacterial translocation $[10,14,19]$. Fungal translocation may be facilitated by upper gastrointestinal bleeding, which is also common in patients with advanced LC [21]. Direct percutaneous inoculation of fungi has been proposed as a route of fungal infection in patients with refractory ascites and a history of paracentesis [19,21]. Bajaj et al. [3] reported that $6.0 \%(8 / 134)$ of fungal infections in patients with LC involved spontaneous peritonitis.

\section{Fungiascites}

A few studies have characterized fungiascites. In a series of nine fungiascites cases, reported by Bucsics et al. [11], seven were ChildPugh class B and two were class C. The in-hospital mortality was $33 \%$ (3/9). Park et al. [27] evaluated 16 cases of spontaneous peritonitis in which Cryptococcus was identified in the ascitic fluid, among which 13 cases (81\%) were reported to involve fungiascites. They also reported that $69 \%(11 / 16)$ of patients with spontaneous peritonitis involving Cryptococcus died within one week after paracenteses [27]. Choi et al. [28] evaluated 21 patients with spontaneous peritonitis in which Candida species (spp.) was identified in the ascitic fluid, among which ten (48\%) were reported to involve fungiascites. Three patients were Child-Pugh class B and seven were class $\mathrm{C}$ and the one-month fungiascites mortality rate was $20 \%(2 / 10)[28]$.

\section{Spontaneous fungal peritonitis}

SFP has mainly been described in patients with end-stage liver disease, such as LC. However, a rare case of SFP due to cardiac origin has been reported [29].

The reported frequencies of culture-positive ascites accompanying SFP have ranged from $0 \%$ to $10 \%[6,10,11,19,23,26]$. It may be difficult to discuss the geographical differences of frequencies of SFP partly because there are limitations in number of clinical studies or number of SFP patients. The review article indicated that frequencies of SFP among culture-positive spontaneous peritonitis ranged 1\%-7\% in studies reported from Asia and Europe [26].

Several previous studies identified Candida albicans as the most frequent SFP isolate $[6,7,9,10,11,14,19,26,30-32]$ and the review article indicated that it is the most frequently isolated pathogen regardless of the geographic differences worldwide [26]. Other isolated fungi included non-albicans Candida (Candida glabrata, Candida krusei, Candida tropicalis, Candida parapsilosis, etc.) [6,26,33], Cryptococcus spp. [10,34,35], Aspergillus spp. [11,21,36], Penicillium spp. [11] and Fusarium solani [37]. In SFP cases, 32\% to $74 \%$ included polymicrobial infections (e.g., bacterial co-colonization) $[6,7,10,11,14,30]$.

The few available SFP studies have identified high Child-Pugh scores (most patients with LC and SFP were Child-Pugh class C $[6,9,10,28])$, high model for end-stage liver disease (MELD) scores [10,26,38], invasive procedures [6], length of hospital stay $[6,30]$ and nosocomial infection as risk factors for SFP occurrence $[4,10,30]$. In previous studies of ascitic culture-positive spontaneous peritonitis diagnosed between 2003 and 2016, nosocomial SFP was confirmed in $7.7 \%(53 / 689)$ of nosocomial spontaneous peritonitis and non-nosocomial SFP was confirmed in 1.7\% (17/1018) of nonnosocomial spontaneous peritonitis [30]. However, further studies may be necessary to identify well-defined risk factors for SFP [4] partly because diagnosis of SFP is often delayed.

\section{Comparative studies between spontaneous peritonitis due to fungus and those due to bacteria}

Lahmer et al. [38] reported no significant differences in prothrombin time (PT) or albumin or creatinine $(\mathrm{Cr})$ levels in patients with LC with SFP and those without peritonitis. They also found that serum bilirubin levels were significantly higher in patients with LC with SFP than in those without peritonitis [38].

A few studies have compared the serum and ascitic fluid findings in SFP and SBP [6-10] or in spontaneous peritonitis with a funguspositive ascitic culture (SFP and fungiascites) and spontaneous peritonitis with a bacteria-positive ascitic culture (culture-positive SBP and bacterascites) [11,12]. Some comparative studies reported no significant differences in PT or albumin or bilirubin levels between SFP and SBP patients [6,7,9] or between spontaneous peritonitis with a fungus-positive ascitic culture and those with a bacteria-positive ascitic culture [11,12]. The reports of serum $\mathrm{Cr}$ level are also inconsistent. As in this series, some comparative studies reported no significant differences in serum $\mathrm{Cr}$ between SFP and SBP $[6,7,10]$ or between spontaneous peritonitis with a fungus-positive ascitic culture and spontaneous peritonitis with a bacteria-positive ascitic culture [11,12]. However, Elkhateeb et al. [9] found that serum Cr was significantly higher in SFP than in SBP (culture-negative neutrocytic ascites).

Regarding the severity of underlying disease, Gravito-Soares et al. [6] reported no significant differences in the Child-Pugh and MELD scores between patients with SFP and SBP. Our previous study [7] also reported no significant differences in the Child-Pugh scores between SFP and culture-positive SBP. Bucsics et al. [11] and our previous study [12] reported no significant differences in the Child-Pugh scores between spontaneous peritonitis with a funguspositive ascitic culture and spontaneous peritonitis with a bacteriapositive ascitic culture. Hwang et al. [10] reported significantly higher Child-Pugh scores in SFP than in SBP.

Regarding the ascitic fluid findings in this series, many studies have observed no significant differences in total protein or albumin between SFP and SBP [6-9] and between spontaneous peritonitis with a fungus-positive ascitic culture and those with a bacteriapositive ascitic culture [12]. 


\section{Prognosis of SFP}

The severity of underlying liver disease [10], a high Child-Pugh [9] or MELD score [14], use of antibacterial prophylaxis [26], presence of hepatorenal syndrome [26], low ascitic fluid protein [26], high acute physiology and chronic health evaluation II score [14] and septic shock have been identified as risk factors associated with inhospital SFP mortality [6].

The in-hospital mortality of SFP reportedly ranges from 33\% to $100 \%[5-7,10,14,19,38]$. Gravito-Soares et al. [6] indicated that 30 -day mortality was $50 \%$ (four of eight patients) in SFP and 24.4\% (29 of 119 patients) in SBP. Similarly, Hwang et al. [10] reported significantly higher one- and six-month mortality rates of $73.3 \%$ (11 of 15 patients) and $93.3 \%$ (14 of 15 patients) in SFP than in SBP $(28.7 \%, 115$ of 401 and $56.1 \%, 225$ of 401 patients, respectively). Our previous study also showed that one- and sixmonth mortality rates were higher in SFP than in culture-positive SBP or bacterascites [7].

Hwang et al. [10] reported that a high SFP mortality rate was related to unresponsiveness to initial empirical treatment for suspected SBP. The condition of nearly all SFP patients has worsened after initial empirical treatment and they have died during the early stage of peritonitis regardless of undergoing antifungal treatment [10]. Some patients with SFP have improved after receiving the initial empirical treatment with no antifungal agents [10]. Those patients may have had SBP and colonization by innocent fungi because it was impossible to distinguish fungal colonization from true SFP in a clinical setting [10].

SFP is usually diagnosed after identifying fungi in cultures of ascitic fluid. The mortality rate is high partly because of delayed diagnosis, lack of clinical signs, lack of suspicion of SFP and delay in treatment with antifungal therapy $[9,10,26,30,38]$. Fungal resistance to empirical specific antifungal therapy together with delayed diagnosis and treatment is related to poor prognosis of SFP [6].

\section{Treatments for SFP}

Echinocandins are recommended as a first-line treatment for patients with LC and nosocomial SFP or critically ill patients with LC and community-acquired SFP [10]. Fluconazole is recommended for less severe infections [26,39]. De-escalation from echinocandins to fluconazole is advised in critically ill patients with LC and SFP when their condition is stable and sensitivity tests are available $[26,39]$.

Although it remains uncertain whether antifungal therapies would decrease the mortality rate associated with spontaneous peritonitis by fungi $[14,30,40]$, early initiation of antifungal therapies is necessary in SFP or suspected SFP cases. However, the questionwhen to start antifungal treatments-remains unanswered partly because of very low-quality of available evidence [4]. Although early differentiation between SFP and SBP may be difficult, clinicians should suspect SFP or SBP due to multidrug resistance bacteria if spontaneous peritonitis has not improved after $48 \mathrm{~h}$ of empirical antibiotic treatment [5]. Therefore, additional administration of antifungal agents (and alternation of antibiotics) may be considered for these patients $[4,5,8]$. Moreover, the recent review article suggests the rapid initiation of antifungal therapy in the presence of septic shock and failure to respond to broad spectrum antibiotic regimen [19]. However, the previous above-mentioned report by
Hwang et al. [10] indicated that the condition of some patients with SFP have worsened after initial empirical antibiotic treatment for SBP, and they have died during the early stage of peritonitis despite undergoing antifungal treatment. Therefore, combination therapies of antibiotics and antifungal agents may be considered for patients with severe LC and nosocomial spontaneous peritonitis, although accumulations of further studies are necessary to justify this.

\section{DECLARATIONS}

There was no funding support and there is no conflict of interests to declare.

I have no relevant financial or nonfinancial relationships to disclose.

\section{REFERENCES}

1. Strauss E. The impact of bacterial infections on survival of patients with decompensated cirrhosis. Ann Hepatol. 2013;13(1):7-19.

2. Chon YE, Kim SU, Lee CK, ParkJY, Kim DY, Han KH, et al. Communityacquired vs. nosocomial spontaneous bacterial peritonitis in patients with liver cirrhosis. Hepatogastroenterology. 2014;61(136):2283-2290.

3. Bajaj JS, Reddy RK, Tandon P, Wong F, Kamath PS, Biggins SW, et al. Prediction of Fungal Infection Development and Their Impact on Survival Using the NACSELD Cohort. Am J Gastroenterol. 2018;113(4):556-563.

4. Maraolo AE, Buonomo AR, Zappulo E, Scotto R, Pinchera B, Gentile I. Unsolved Issues in the Treatment of Spontaneous Peritonitis in Patients with Cirrhosis: Nosocomial Versus Community-acquired Infections and the Role of Fungi. Rev Recent Clin Trials. 2019;14(2):129-135.

5. Fiore M, Maraolo AE, Leone S, Gentile I, Cuomo A, Schiavone V, et al. Spontaneous peritonitis in critically ill cirrhotic patients: a diagnostic algorithm for clinicians and future perspectives. Ther Clin Risk Manag. 2017;13:1409-1414.

6. Gravito-Soares M, Gravito-Soares E, Lopes S, Ribeiro G, Figueiredo P. Spontaneous fungal peritonitis: a rare but severe complication of liver cirrhosis. Eur J Gastroenterol Hepatol. 2017;29(9):1010-1016.

7. Shizuma T. Retrospective Comparison of Spontaneous Fungal and Spontaneous Bacterial Peritonitis in Patients with Liver Cirrhosis. Sylwan. 2018;162(4):15-32.

8. Alexopoulou A, Vasilieva L, Agiasotelli D, Dourakis SP. Fungal infections in patients with cirrhosis. J Hepatol. 2015;63(4):1043-1045.

9. Elkhateeb SR, Gouda NS, Ibrahim AS, Anwar R, Eissa LA. PCRdetected fungal infection is associated with fatal outcomes incirrhotic patients with spontaneous peritonitis. Egyptian J Basic Appl Sciences. 2017;4(1):42-46.

10.Hwang SY, Yu SJ, Lee JH, Kim JS, Yoon JW, Kim YJ, et al. Spontaneous fungal peritonitis: a severe complication in patients with advanced liver cirrhosis. Eur J Clin Microbiol Infect Dis. 2014;33(2):259-264.

11. Bucsics T, Schwabl P, Mandorfer M, Peck-Radosavljevic M. Prognosis of cirrhotic patients with fungiascites and spontaneous fungal peritonitis (SFP). J Hepatol. 2016;64(6):1452-1454.

12. Shziuma T. Retrospective Comparative Study of Spontaneous Peritonitis Associated with Fungal and Bacterial Infection in Patients with Liver Cirrhosis. J Liver. 2018;7(1):226.

13.Rimola A, García-Tsao G, Navasa M, Piddock LJ, Planas R, Bernard B, et al. Diagnosis, treatment and prophylaxis of spontaneous bacterial peritonitis: a consensus document. International Ascites Club. J Hepatol. 2000;32(1):142-153. 
14. Bremmer DN, Garavaglia JM, Shields RK. Spontaneous fungal peritonitis: a devastating complication of cirrhosis. Mycoses. 2015; 58(7):387-393.

15.Gupta P, Ahmad A, Khare V, Kumar A, Banerjee G, Verma N, et al. Comparative evaluation of pan-fungal real-time PCR, galactomannan and (1-3)- $\beta$-D-glucan assay for invasive fungal infection in paediatric cancer patients. Mycoses. 2017;60(4):234-240.

16. Khoury N, Amit S, Geffen Y, Adler A. Clinical utility of pan-microbial PCR assays in the routine diagnosis of infectious diseases. Diagn Microbiol Infect Dis. 2019;93(3):232-237.

17. El-Mahallawy HA, Shaker HH, Ali Helmy H, Mostafa T, Razak AboSedah A. Evaluation of pan-fungal PCR assay and Aspergillus antigen detection in the diagnosis of invasive fungal infections in high risk paediatric cancer patients. Med Mycol. 2006;44(8):733-739.

18.Shabaan AE, Elbaz LM, El-Emshaty WM, Shouman B. Role of serum $(1,3)-\beta$-d-glucan assay in early diagnosis of invasive fungal infections in a neonatal intensive care unit. J Pediatr (Rio J). 2018;94(5):559-565.

19.Tariq T, Irfan FB, Farishta M, Dykstra B, Sieloff EM, Desai AP. Spontaneous fungal peritonitis: Micro-organisms, management and mortality in liver cirrhosis-A systematic review. World J Hepatol. 2019;11(7):596-606.

20.Shizuma T. Possible Laboratory Markers for Diagnosing Spontaneous Bacterial Peritonitis: A Literature Review. Sylwan. 2017;161(6):157-180.

21. Hassan EA, Abd El-Rehim AS, Hassany SM, Ahmed AO, Elsherbiny NM, Mohammed MH. Fungal infection in patients with end-stage liver disease: low frequency or low index of suspicion. Int J Infect Dis. 2014;23:69-74.

22.Zhao H, Gu X, Zhao R, Shi Y, Sheng J. Evaluation of prognostic scoring systems in liver cirrhosis patients with bloodstream infection. Medicine (Baltimore). 2017;96(50):e8844.

23.Shizuma T. Spontaneous bacterial and fungal peritonitis in patients with liver cirrhosis: A literature review. World J Hepatol. 2018;10(2):254266.

24.Alexopoulou A, Agiasotelli D, Vasilieva LE, Dourakis SP. Bacterial translocation markers in liver cirrhosis. Ann Gastroenterol. 2017;30(5):486-497.

25. Guarner C, Soriano G. Bacterial translocation and its consequences in patients with cirrhosis. Eur J Gastroenterol Hepatol. 2005;17(1):27-31.

26. Fiore M, Leone S. Spontaneous fungal peritonitis: Epidemiology, current evidence and future prospective. World J Gastroenterol. 2016;22(34):7742-7747.

27. Park WB, Choe YJ, Lee KD, Lee CS, Kim HB, Kim NJ, et al. Spontaneous cryptococcal peritonitis in patients with liver cirrhosis. Am J Med. 2006;119(2):169-171.
28. Choi SH, Soo Kim Y, Chung JW, Choo EJ, Kwak YG, Lee YS, et al. Clinical significance of untreated Candida species isolated from ascites in cirrhotic patients. Scand J Infect Dis. 2004;36(9):649-655.

29.Wang Y, Gandhi S, Attar BM. Spontaneous fungal peritonitis in ascites of cardiac origin. ACG Case Rep J. 2017;4(1):e42.

30.Fiore M, Chiodini P, Pota V, Sansone P, Passavanti MB, Leone S, et al. Risk of spontaneous fungal peritonitis in hospitalized cirrhotic patients with ascites: a systematic review of observational studies and metaanalysis. Minerva Anestesiol. 2017;83(12):1309-1316.

31. Friedrich K, Nüssle S, Rehlen T, Stremmel W, Mischnik A, Eisenbach C. Microbiology and resistance in first episodes of spontaneous bacterial peritonitis: implications for management and prognosis. J Gastroenterol Hepatol. 2016;31(6):1191-1195.

32.Karvellas CJ, Abraldes JG, Arabi YM, Kumar A. The Cooperative Antimicrobial Therapy of Septic Shock (CATSS) Database Research Group. Appropriate and timely antimicrobial therapy in cirrhotic patients with spontaneous bacterial peritonitis-associated septic shock: a retrospective cohort study. Aliment Pharmacol Ther. 2015;41(8):747757.

33.Shi L, Wu D, Wei L, Liu S, Zhao P, Tu B, et al. Corrigendum: Nosocomial and Community-Acquired Spontaneous Bacterial Peritonitis in patients with liver cirrhosis in China: Comparative Microbiology and Therapeutic Implications. Sci Rep. 2017;7(1):46868.

34.Bal CK, Bhatia V, Khillan V, Rathor N, Saini D, Daman R, et al. Spontaneous cryptococcal peritonitis with fungemia in patients with decompensated cirrhosis: Report of two cases. Indian J Crit Care Med. 2014;18(8):536-539.

35.Albert-Braun S, Venema F, Bausch J, Hunfeld KP, Schäfer V. Cryptococcus neoformans peritonitis in a patient with alcoholic cirrhosis: case report and review of the literature. Infection. 2005;33(4):282-288.

36.Jindal A, Kumar M, Bhadoria AS, Maiwall R, Sarin SK. A randomized open label study of 'imipenem vs. cefepime' in spontaneous bacterial peritonitis. Liver Int. 2016;36(5):677-687.

37. Mayr U, Rasch S, Schmid RM, Huber W, Lahmer T. First description of spontaneous fungal peritonitis caused by Fusarium solani in a critically ill patient with liver cirrhosis. New Microbes New Infect. 2017;20:16-17.

38.Lahmer T, Brandl A, Rasch S, Schmid RM, Huber W. Fungal Peritonitis: Underestimated Disease in Critically Ill Patients with Liver Cirrhosis and Spontaneous Peritonitis. PLoS One. 2016;11(7):e0158389.

39.Fiore M, Leone S. Use of antifungals in critically ill cirrhotic patients with spontaneous peritonitis. J Hepatol. 2016;64(4):986-987.

40.Dubler S, Laun M, Koch C, Hecker A, Weiterer S, Siegler BH, et al. The impact of real life treatment strategies for Candida peritonitis-A retrospective analysis. Mycoses. 2017;60(7):440-446. 